\title{
Telephone based Interventions for Psychological Problems in Hospital Isolated Patients with COVID-19
}

\author{
Ju-Wan Kim ${ }^{1}$, Robert Stewart ${ }^{2,3}$, Seung-Ji Kang ${ }^{4}$, Sook-In Jung ${ }^{4}$, Sung-Wan Kim ${ }^{1}$, Jae-Min Kim \\ ${ }^{1}$ Department of Psychiatry, Chonnam National University Medical School, Gwangju, Korea, ${ }^{2}$ King's College London, Institute of Psychiatry, \\ Psychology and Neuroscience, ${ }^{3}$ South London and Maudsley NHS Foundation Trust, London, UK, ${ }^{4}$ Department of Infectious Diseases Medicine, \\ Chonnam National University Medical School, Gwangju, Korea
}

Objective: The COVID-19 is overwhelming health care systems globally. Hospital isolation may generate considerable psychological stress. However, there has been scarce evidence on psychological interventions for these patients due to maintain staff safety. We investigated the feasibility and effectiveness of telephone based interventions for psychological problems in hospital isolated patients with COVID-19.

Methods: Psychiatrists visited the ward where the patients were hospitalized and interventions were given by using a ward telephone for 30 minutes. All patients were approached to receive a two-week psychological intervention program and/or pharmacotherapy whenever needed. Psychological problems were assessed at baseline, one, and two weeks. For the assessment of anxiety and depressive symptoms, the Hospital Anxiety and Depression Scale was administered to patients once a week. Insomnia severity index and Beck Depression Inventory 9 item were checked weekly to assess insomnia and suicide idea.

Results: Of 33 enrolled, clinically meaningful psychological symptoms were found in 6 (18\%) patients for anxiety; 13 $(39 \%)$ for depression; 10 (30\%) for insomnia; and 3 (9\%) for suicidal ideation. In 9 patients (27\%), psychotropic medications were prescribed to manage anxiety, agitation, depressed mood, insomnia, impulsivity, and suicide idea. Compared to baseline, significant improvements were found in anxiety, depression, and suicidal ideation at one week. There were no statistical differences between the values evaluated at baseline and at two weeks.

Conclusion: Our report at least indicates potential usefulness of telephone based interventions in hospital isolated patients with COVID-19, and will hopefully form the basis for future randomized clinical trials.

KEY WORDS: COVID-19; Depression; Anxiety; Suicide.

\section{INTRODUCTION}

The COVID-19, the illness caused by severe acute respiratory coronavirus 2 (SARS-CoV-2), is overwhelming health care systems globally. As of April 28, 2020, South Korea confirmed 10,752 cases and 244 deaths after the first outbreak on January 20. Since targeted medications

Received: May 4, 2020 / Revised: June 16, 2020

Accepted: June 19, 2020

Address for correspondence: Jae-Min Kim

Department of Psychiatry, Chonnam National University Medical

School, 160 Baekseo-ro, Dong-gu, Gwangju 61469, Korea

E-mail: jmkim@chonnam.ac.kr

ORCID: https://orcid.org/0000-0001-7409-6306

Sung-Wan Kim

Department of Psychiatry, Chonnam National University Medical School, 160 Baekseo-ro, Dong-gu, Gwangju 61469, Korea

E-mail: swkim@chonnam.ac.kr

ORCID: https://orcid.org/0000-0002-6739-2163 against COVID-19 have not been developed, conventional therapeutic approaches are hospital isolation and/or symptomatic care. The hospital isolation may bring out considerable psychological problems to the patients. Even quarantine due to potentially being exposed to contagious diseases had negative psychological impact crosssectionally and longitudinally [1]. However, there has been scarce evidence on psychological interventions for these patients. After the COVID-19 outbreak, the importance of psychological well-being has been stressed in considerably many article, while there have been no data driven evidences of the psychological interventions. This probably due to the facts of prohibiting contact from infectious patients and being given priority to medical care. Recent research has focused on the use of online or telephonic psychological interventions to reduce the risk of

(c) This is an Open-Access article distributed under the terms of the Creative Commons Attribution Non-Commercial License (http://creativecommons.org/licenses/by-nc/4.0) which permits unrestricted non-commercial use, distribution, and reproduction in any medium, provided the original work is properly cited. 
infection-related emotional distress [2]. However, there is insufficient evidence of their effectiveness and studies of COVID-19 in this regard have not yet been published. In this article, we investigated the feasibility and effectiveness of telephone based interventions for psychological problems in hospital isolated patients with COVID-19.

\section{METHODS}

Participants were consecutively recruited from all hospitalized patients with COVID-19 at the Department of Infectious Diseases of Chonnam National Univeristy Bitgoeul Hospital (CNUBH), Gwangju, South Korea. All patients were approached to receive a two-week psychological intervention program and/or pharmacotherapy whenever needed (detailed in Table 1). Study psychiatrists visited the ward where the patients were hospitalized and interventions were given by using a ward telephone for 30 minutes. Before and after the interventions, patients' information was shared between the psychiatrists and medical treatment team to facilitate collaborative care. The baseline intervention was initiated at a median 3 days after hospitalization, and was then repeated twice a week for two weeks (maximum five) or to discharge, if earlier. Psychotropic medications were prescribed if needed. Patients followed for at least one week were included for analysis. The first patient was enrolled on February 29, 2020 and the last patient completed the 2-week follow-up evaluation on April 13, 2020. Psycholo- gical problems were assessed at baseline, and one and two weeks thereafter. Anxiety and depression were evaluated by Hospital Anxiety and Depression Scale-anxiety subscale (HADS-A) and -depression subscale (HADS-D) [3]; and insomnia by Insomnia severity index (ISI) [4] all of which considered as clinically meaningful when scoreed $8+$. Suicidal ideation was recorded by item 9 of Beck Depression Inventory (BDI) [5], considered as being present when score $1+$. As shown in Table 1, we used a semi-structured interview protocol. Therapists adopted a primarily supportive approach to treat patients' anxiety, depression, and insomnia [6]. Indeed, as a non-face-to-face intervention, telephone sessions should focus more on providing psychoeducation for the isolated patient to produce short-term effects. For example, beginning at admission, patients should be offered sufficient information about the psychological effects of infectious diseases and isolation to reduce excessive fear. Therapists could also correct inappropriate cognitive appraisals to reduce distress. For example, one older patient thought she would die from COVID-19 and was not actively involved in the medical treatment. The therapist adopted a cognitive-behavioral approach to this misunderstanding of the disease, and the patient was discharged with a good prognosis. Data on follow-ups were compared to those on baseline by Wilcoxon signed-rank test or binomial exact test, as appropriate. Approval was provided by the CNUH Institutional Review Board (CNUH-2020-100). Written informed consent was waived in light of the urgent need to collect

Table 1. Telephone based interventions for COVID-19

\begin{tabular}{|c|c|}
\hline Objectives & $\begin{array}{l}\text { Reducing psychological symptoms and improving adaptation to the ward environment by providing education, } \\
\text { empathy, encouragement, reassurance and suggestion to isolated patients with COVID-19 }\end{array}$ \\
\hline $\begin{array}{l}\text { Baseline } \\
\text { evaluation }\end{array}$ & $\begin{array}{l}\text { - Formation of therapeutic alliance: Providing a sense of care and support through empathy for COVID-19 infection } \\
\text { and hospital isolation } \\
\text { - Providing mental health guideline for isolation/quarantine } \\
\text { - Psychological assessment } \\
\text { - Reassurance of excessive fear about COVID-19 } \\
\text { - Prescribing psychotropic medication }\end{array}$ \\
\hline $\begin{array}{l}\text { Evaluation until } \\
2 \text { weeks }\end{array}$ & $\begin{array}{l}\text { - Formation of therapeutic alliance: Providing a sense of care and support through empathy for COVID-19 infection } \\
\text { and hospital isolation } \\
\text { - Symptom follow-up and psychological assessment } \\
\text { - Consolation and encouragement for COVID-19 diagnostic test result } \\
\text { - Active listening and suggestion of ward adaptation difficulties/stress factor } \\
\text { - Cognitive reconstructions for irrational beliefs } \\
\text { - Prescribing psychotropic medication }\end{array}$ \\
\hline Before discharge & $\begin{array}{l}\text { - Reassurance and guidance for fear of re-infection } \\
\text { - Advice of psychological problems that may be experienced after discharge }\end{array}$ \\
\hline
\end{tabular}


data and minimize the risk of infection.

\section{RESULTS}

Of 50 patients hospitalized during the study period, 8 could not be approached due to medical conditions. Of remaining 42, 6 (14\%) refused to participate. Of 36 enrolled, 3 were discharged before 1 week, hence 33 comprised the study sample. Their mean age was 45 years (standard deviation $[\mathrm{SD}], \pm 18.34$ ). Any patient withdrawn from the intervention after enrollment (Supplementary Fig. 1; available online). The mean duration of hospitalization for patients was 19.6 days (SD, \pm 7.23 ). At baseline, clinically meaningful psychological symptoms were found as follows: anxiety in $6(18 \%)$ patients; depression in $13(39 \%)$; insomnia in $10(30 \%)$; and suicidal ideation in $3(9 \%)$. Individual patient data are summarized in

A Hospital anxiety and depression scale-anxiety subscale

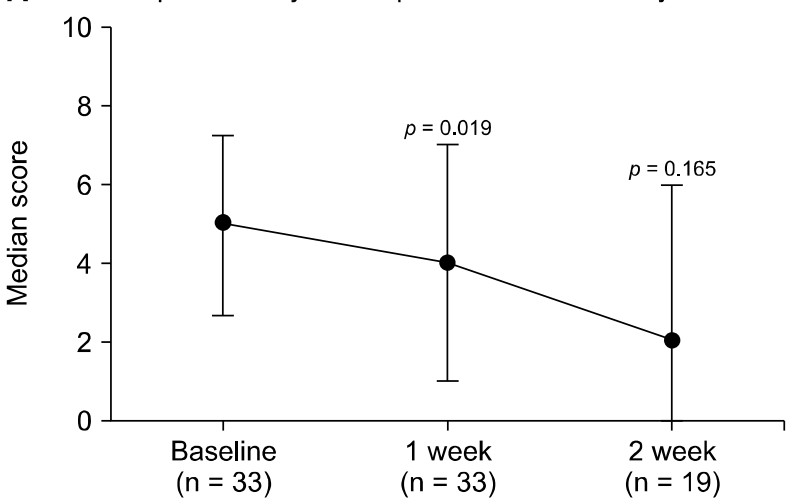

C

Insomnia severity index

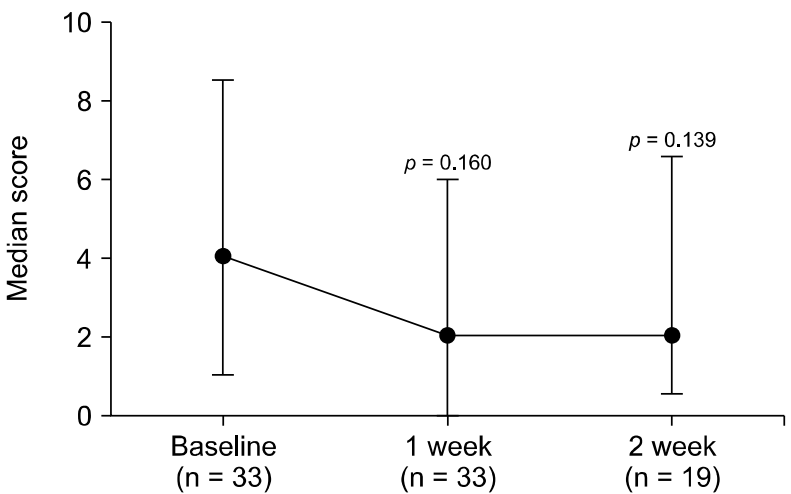

Supplementary Table 1 (available online). In 9 patients $(27 \%)$, psychotropic medications including mirtazapine, escitalopram, quetiapine, trazodone, and benzodiazepines were prescribed to manage anxiety, agitation, depressed mood, insomnia, impulsivity, and suicide idea. Seven patients were treated by lopinavir, hydroxycholoroquine, and interferon to treat COVID-19. However, severities of depressed mood, anxiety and insomnia were not statistically significantly different compared to patients without those treatments. Values are displayed and compared in Figure 1 using medians and interquartile ranges, or number $(\%)$. Compared to baseline, significant improvements were found in anxiety, depression, and suicidal ideation at one week. Fourteen patients were discharged before two weeks. There were no statistical differences between the values evaluated at baseline and at two weeks.

B Hospital anxiety and depression scale-depression subscale

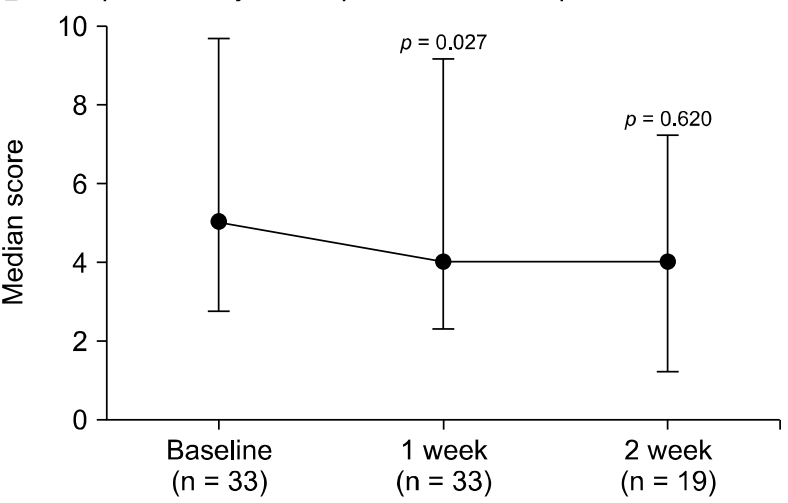

D Beck depression inventory item 9 (suicidal ideation) $\geq 1$

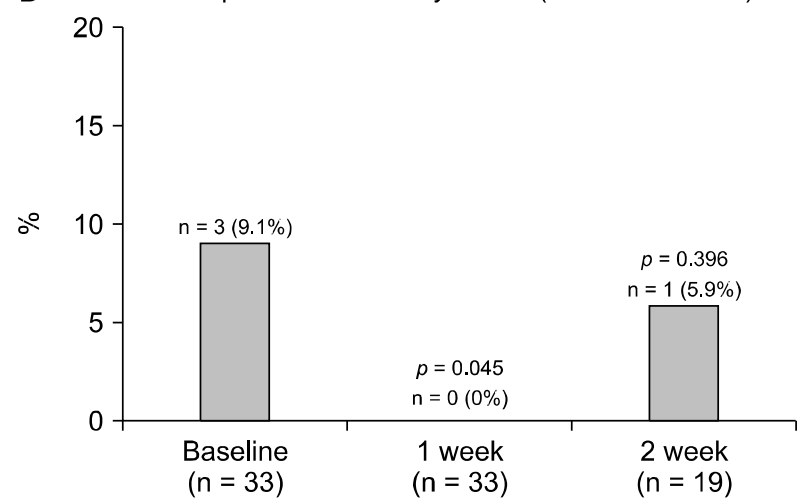

Fig. 1. Changes in anxiety, depression, insomnia, and suicidal ideation from baseline to week 1 and 2. (A, B) The Hospital Anxiety and Depression Scale-anxiety subscale and -depression subscale consist of 7 items with a total score ranging from 0 to 28, each subscale score of 8 or above was used as the cut-off point indicating a certain level of significant distress. (C) The Insomnia Severity Index consist of 7 question assessing the impact of insomnia with a total score ranging from 0 to 28 , with a score of 8 or above was used as the cut-off point indicating a certain level of insomnia. (D) The item 9 of Beck Depression Inventory, which addresses suicidal thoughts or wishes, has a four-level response set including. Suicidal ideation was considered as being present when score 1 or above. $p$ values were drawn by comparing between data on follow-ups and baseline by Wilcoxon signed-rank test or binomial exact test, as appropriate. Error bars indicate interuquartile ranges. 


\section{DISCUSSION}

Psychological problems were frequent in hospital isolated patients with COVID-19. Telephone based interventions were highly acceptable in these patients: $14 \%$ refusal rate for enrollment and $0 \%$ withdrawal during the study period. Furthermore, it had rapid and beneficial effects on reducing anxiety, depression, and suicidal ideation for one week. These effects could be explained in several ways. First, to the hospital isolated patients, being given sufficient information on possible psychological distress and emotional support may reduce excessive fear and promote a sense of stability. First, fear, which is a common experience for isolated and/or quarantined patients with infectious diseases [7], may be exacerbated by insufficient information. Second, there is sufficient evidence in support of the use of telemedicine for the delivery of mental health services. According to a recent review article, psychological interventions delivered via telepsychiatry and face-to-face sessions resulted in similar treatment outcomes [8]. However, six of 33 patients showed significantly worsened psychological symptoms during the isolation, which is probably attributable to the short treatment period. According to current treatment guidelines, treatment responses can be fully evaluated 612 weeks after confirmed treatment initiation $[9,10]$. However, because patients in this study were discharged when COVID-19 was adequately treated, we were unable to deliver our intervention for a sufficient period of time. Therefore, continued post-discharge psychological evaluation and treatment are needed. One important limitation of this study was our small sample, which precluded evaluations of the effects of psychiatric history, gender, and quarantine period on psychological changes. These issues warrant additional, larger studies. In conclusion, the generalizability of the findings was limited due to one arm study design, small sample size, and being conducted in a single center. However, the present report could suggest usefulness of telephone based interventions in hospital isolated patients with infectious diseases [11], and may serve grounds for future randomized clinical trials or long-term outcomes studies [12].

\section{- Acknowledgments}

The study was funded by a grant of National Research Foundation of Korea Grant (NRF- 2019M3C7A1031345), and was supported by Basic Science Research Program through the National Research Foundation of Korea (NRF) funded by the Ministry of Science, ICT and future Planning (NRF- 2020R1A2C2003472). RS is part-funded by the National Institute for Health Research (NIHR) Biomedical Research Centre at the South London and Maudsley NHS Foundation Trust and King's College London.

\section{Conflicts of Interest}

No potential conflict of interest relevant to this article was reported.

\section{Author Contributions}

Ju-Wan Kim and Jae-Min Kim had full access to all of the data in the study and take responsibility for the integrity of the data and the accuracy of the data analysis. Study concept and design: Sung-Wan Kim, Jae-Min Kim. Acquisition of data: Ju-Wan Kim, Seung-Ji Kang, Sook-In Jung. Analysis and interpretation of data: Ju-Wan Kim, Robert Stewart, Sung-Wan Kim, Jae-Min Kim. Drafting of the manuscript: Ju-Wan Kim, Robert Stewart, Sung-Wan Kim, Jae-Min Kim. Critical revision of the manuscript for important intellectual content: Ju-Wan Kim, Robert Stewart, Seung-Ji Kang, Sook-In Jung, Sung-Wan Kim, Jae-Min Kim. Statistical analysis: Ju-Wan Kim, Robert Stewart, Sung-Wan Kim, Jae-Min Kim. Obtained funding: Ju-Wan Kim. Administrative, technical, or material support: Ju-Wan Kim, Robert Stewart, Sung-Wan Kim, Jae-Min Kim. Study supervision: Sung-Wan Kim, Jae-Min Kim.

\section{- ORCID}

Ju-Wan Kim https://orcid.org/0000-0002-9888-1090 Robert Stewart https://orcid.org/0000-0002-4435-6397 Seung-Ji Kang https://orcid.org/0000-0002-3298-0520 Sook-In Jung Sung-Wan Kim https://orcid.org/0000-0002-1577-678X Jae-Min Kim https://orcid.org/0000-0002-6739-2163 https://orcid.org/0000-0001-7409-6306

\section{REFERENCES}

1. Brooks SK, Webster RK, Smith LE, Woodland L, Wessely S, Greenberg N, et al. The psychological impact of quarantine and how to reduce it: rapid review of the evidence. Lancet 2020;395:912-920.

2. Corruble E. A viewpoint from Paris on the COVID-19 pandemic: a necessary turn to telepsychiatry. J Clin Psychiatry 
2020;81:20com13361.

3. Zigmond AS, Snaith RP. The hospital anxiety and depression scale. Acta Psychiatr Scand 1983;67:361-370.

4. Bastien $\mathrm{CH}$, Vallières $\mathrm{A}$, Morin $\mathrm{CM}$. Validation of the Insomnia Severity Index as an outcome measure for insomnia research. Sleep Med 2001;2:297-307.

5. Beck AT, Ward CH, Mendelson M, Mock J, Erbaugh J. An inventory for measuring depression. Arch Gen Psychiatry 1961; 4:561-571.

6. Novalis PN, Rojcewicz SJ Jr, Peele R. Clinical manual of supportive psychotherapy. Washington, D.C.:American Psychiatric Press; 1993.

7. Rubin GJ, Harper S, Williams PD, Öström S, Bredbere S, Amlôt $R$, et al. How to support staff deploying on overseas humanitarian work: a qualitative analysis of responder views about the 2014/15 West African Ebola outbreak. Eur J Psychotraumatol 2016;7:30933.

8. Hubley S, Lynch SB, Schneck C, Thomas M, Shore J. Review of key telepsychiatry outcomes. World I Psychiatry 2016;6: 269-282.
9. Bauer M, Whybrow PC, Angst J, Versiani M, Möller HJ; World Federation of Societies Biological Psychiatry Task Force on Treatment Guidelines for Unipolar Depressive Disorders. World Federation of Societies of Biological Psychiatry (WFSBP) guidelines for biological treatment of unipolar depressive disorders, part 1: acute and continuation treatment of major depressive disorder. World J Biol Psychiatry 2002;3:5-43.

10. Malhi GS, Outhred T, Morris G, Boyce PM, Bryant R, Fitzgerald PB, et al. Royal Australian and New Zealand College of Psychiatrists clinical practice guidelines for mood disorders: bipolar disorder summary. Med J Aust 2018;208: 219-225.

11. Kim SW, Su KP. Using psychoneuroimmunity against COVID-19. Brain Behav Immun 2020;87:4-5.

12. Kim JM, Stewart R, Lee YS, Lee HJ, Kim MC, Kim JW, et al. Effect of escitalopram vs placebo treatment for depression on long-term cardiac outcomes in patients with acute coronary syndrome: a randomized clinical trial. JAMA 2018;320:350358. 